PROCEEDINGS OF THE

AMERICAN MATHEMATICAL SOCIETY

Volume 102, Number 4, April 1988

\title{
ON THE JACOBSON RADICAL OF SOME ENDOMORPHISM RINGS
}

\author{
MANFRED DUGAS
}

(Communicated by Bhama Srinivasan)

\begin{abstract}
In this note we deal with a question raised by R. S. Pierce in 1963: Determine the elements of the Jacobson radical of the endomorphism ring of a primary abelian group by their action on the group. We concentrate on separable abelian $p$-groups and give a counterexample to a conjecture of $\mathrm{A}$. D. Sands. We also show that the radical can be pinned down if the endomorphism ring is a split-extension of its ideal of all small maps.
\end{abstract}

Introduction. All groups in this note are abelian $p$-groups for some fixed but arbitrary prime $p$. Our notations are standard as in $[\mathbf{F}]$. It is known that the endomorphism ring $\operatorname{End}(A)$ of an abelian $p$-group $A$ determines the group up to isomorphism. R. Pierce $[\mathbf{P}]$ raised the question of describing the Jacobson radical $J(\operatorname{End}(A))$ of $\operatorname{End}(A)$ by its action on the group. This problem was solved by W. Liebert $[\mathbf{L}]$, J. Hausen $[\mathbf{H}]$ and Hausen-Johnson $[\mathbf{H J}]$ for $\Sigma$-cyclic, torsioncomplete and sufficiently projective $p$-groups. (For a separable $p$-group sufficiently projective is the same as $\omega_{1}$-separable.) If $A$ is a (separable) p-group, let $H(A)=$ $\{\varphi \in \operatorname{End}(A)|| x|<| x \varphi \mid$ for all $0 \neq x \in A[p]\}$ be the ideal of all maps acting height increasing on the socle of $A$, and let $C(A)$ be the ideal of all elements of End $(A)$ mapping each Cauchy sequence in $A[p]$ onto a convergent one. (For $x \in A$, $|x|$ denotes the $p$-height of $x$ in $A$ and topological notations refer to the $p$-adic topology.) If $A$ is torsion-complete, $J(\operatorname{End}(A))=H(A)$, if $A$ is $\Sigma$-cyclic or $\omega_{1-}$ separable, $J(\operatorname{End}(A))=H(A) \cap C(A)$, and $H(A) \cap C(A) \subset J(\operatorname{End}(A))$ for all separable $p$-groups (cf. $[\mathbf{S}])$. The purpose of this paper is to show that $J(\operatorname{End}(A))$ is in general not equal to $C(A) \cap H(A)$ for separable $p$-groups $A$. We will use that $J(\operatorname{End}(A)) \cap E_{s}(A)=E_{s}(A) \cap H(A)$, where $E_{s}(A)$ is the ideal of all small endomorphisms of $A$ (cf. $[\mathbf{S}]$ ). Recently, many complicated $p$-groups have been constructed in [DG1, DG2, CG]. All these groups enjoy the property that $\operatorname{End}(A)$ is a split extension of $E_{s}(A)$, i.e. $\operatorname{End}(A)=R \oplus E_{s}(A)$ for some subring $R$ of $\operatorname{End}(A)$. The way these groups are constructed, $R \cap H(A)=p R$ and $\bar{H}_{R}(A)=\bar{H}_{R}^{*}(A)$, i.e. if $r \in R-H(A)$, then for all $n$ there is $0 \neq x \in p^{n} A[p]$ such that $x$ and $x r$ have the same height. In this situation Theorem 1 below implies

$$
J(\operatorname{End}(A))=(J(R) \cap H(A)) \oplus\left(E_{s}(A) \cap H(A)\right)
$$

and we have $J(\operatorname{End}(A))=H(A) \cap C(A)$ for these groups. We will construct a ring $R$ and use the realization result in $[\mathbf{C}]$ to obtain a separable $p$-group $A$ such that

Received by the editors July 17, 1986 and, in revised form, December 18, 1986.

1980 Mathematics Subject (Classification (1985 Revision). Primary 20K10, 20K30.

Key words and phrases. Abelian p-group, endomorphism ring, small endomorphisms, Jacobson radical, height-preserving maps. 
$\operatorname{End}(A)=R \oplus E_{s}(A)$ and $(J(R)-p R) \cap H(A)$ and $(R-J(R)) \cap H(A)$ are both not empty. Since $C(A) \cap R=p R$ for this group $A$, we have that $H(A) \cap C(A)$ is a proper subset of $J(\operatorname{End}(A))$. This makes it hard to believe that Pierce's question has a positive answer for all separable $p$-groups.

The construction. Let $A$ be a separable $p$-group and $E=\operatorname{End}(A)$ the endomorphism ring of $A$. If $S \subset E$, and $U \subset A[p]$ are subsets, define $\bar{H}_{S}(A, U)=\{\varphi \in$ $S|\exists 0 \neq x \in U| x,|=| x \varphi \mid\}$. Observe that $\bar{H}_{E}(A, U) \cap S=\bar{H}_{S}(A, U)$ and if $U \subset V$ we have $\bar{H}_{S}(A, U) \subset \bar{H}_{S}(A, V)$. We also define $H(A)=E-\bar{H}_{E}(A, A[p])$, $\bar{H}_{S}(A)=\bar{H}_{S}(A, A[p])$ and $\bar{H}_{S}^{*}(A)=\bigcap_{n} \bar{H}_{S}\left(A, p^{n} A[p]\right)$. Moreover, let $H_{S}(A)=$ $S \cap H(A)$ and $H_{S}(A, U)=S-\bar{H}_{S}(A, U)$.

THEOREM 1. Let $A$ be a separable $p$-group such that $E=E(A)=R \oplus E_{s}(A)$ is a split extension of $E_{s}(A)$ and $\bar{H}_{R}(A)=\bar{H}_{R}^{*}(A)$. Then $J(E)=H_{J(R)}(A) \oplus$ $H_{E_{s}(A)}(A)$.

Proof. Let $r \in R$, and $\sigma \in E_{s}(A)$ with $r+\sigma \in J(E)$. Then $(r+\sigma) t$ is right quasi-regular for all $t \in R$ and, since $E=R \oplus E_{s}(A)$, the element $r t$ is right quasiregular in $R$ as well as $r \in J(R)$. Now suppose $r \notin H_{J(R)}(A)$. Since $\sigma$ is small and $\bar{H}_{R}(A)=\bar{H}_{R}^{*}(A)$, we find $n<\omega$ and $0 \neq x \in p^{n} A[p]$ such that $x \sigma=0$ and $|x|=|x r|$. Let $F$ be a finite summand of $A$ containing $x r$ and let $\rho: A \rightarrow F$ be the natural projection. Then $x r=x r \rho$ and $|x|=|x r|=|x r \rho|=|x(r+\sigma) \rho|$. Since $A \rho=F$ is finite, $\rho \in E_{s}(A)$ and hence $(r+\sigma) \rho \in J(E) \cap E_{s}(A)=E_{s}(A) \cap H(A)$ (cf. $[\mathbf{S}])$. This contradicts the above equation of heights and we conclude $r \in H_{J(R)}(A)$. Now let $t \in R, \sigma \in E_{s}(A)$ and $r \in H_{J(R)}(A)$. Then there is $s \in R$ such that $(1-r t) s=1$. This implies $(1-r(t+\sigma)) s=(1-r t) s-r \sigma s=1-r \sigma s$. Since $\sigma \in E_{s}(A)$ and $r \in H_{J(R)}(A)$ we have that $r \sigma s \in H_{E_{s}(A)}(A) \subset J(E)$ and there is $\tau \in E$ with $(1-r \sigma s) \tau=1$. This implies $(1-r(t+\sigma)) s \tau=1$ and $r \in J(E)$. We obtain $H_{J(R)}(A) \subset J(E) \subset H_{J(R)} \oplus E_{s}(A)$ which together with $J(E) \cap E_{s}(A)=H_{E_{s}(A)}(A)$ implies the desired equation.

We now construct our ring:

Let $\omega$ be the set of natural numbers including 0 and let

$$
B=\bigoplus_{i \in \omega}\left\langle f_{i}\right\rangle \oplus \bigoplus_{i \in \omega}\left\langle g_{i}\right\rangle \oplus \bigoplus_{i \in \omega}\left\langle h_{i}\right\rangle
$$

be a $\Sigma$-cyclic $p$-group with $\exp \left(f_{i}\right)=i+1=\exp \left(g_{i}\right)$ and $\exp \left(h_{i}\right)=i+2$. We define elements $\alpha, \beta, \gamma \in \operatorname{End}(B)$ by setting $f_{i} \alpha=p f_{i+1}, f_{i} \beta=g_{i}$ and $f_{i} \gamma=p h_{i}$, and $\alpha$, $\beta$ and $\gamma$ are 0 on the $g_{i}$ 's and $h_{i}$ 's. Let $S=\langle 1, \alpha, \beta, \gamma\rangle$ be the subring of $\operatorname{End}(B)$ generated by these elements and $R=\widehat{S}$ be the $p$-adic completion of $S$. We have the following relations:

(1) $\beta \alpha=\gamma \alpha=\beta^{2}=\gamma^{2}=\beta \gamma=\gamma \beta=0$.

Each element $r \in S$ has a unique representation:

(2) $r=\sum_{i=0}^{n} \alpha^{i} a_{i}+\sum_{i=0}^{m} \alpha^{i} \beta b_{i}+\sum_{i=0}^{k} \alpha^{i} \gamma c_{i}$ with $a_{i}, b_{i}$ and $c_{i}$ integers.

Therefore each element $x \in R=\widehat{S}$ has a unique representation:

(3) $x=\sum_{i=0}^{\infty} \alpha^{i} a_{i}+\sum_{i=0}^{\infty} \alpha^{i} \beta b_{i}+\sum_{i=0}^{\infty} \alpha^{i} \gamma c_{i}$ where $\left\{a_{i}\right\},\left\{b_{i}\right\}$ and $\left\{c_{i}\right\}$ are $p$-adic zero-sequences in $J_{p}$, the ring of $p$-adic integers. Let $I$ be the set of all $x \in R$ with all $a_{i}$ 's being 0 . This is the ideal of $R$ generated by $\beta$ and $\gamma$. An easy computation shows: 
(4) Let $x \in R$ be as in (3). Then $\exp \left(f_{k} x\right)=\max _{i \in \omega}\left\{k+1-\left|a_{i}\right|, k+1-\left|b_{i}\right|\right.$, $\left.k+1-\left|c_{i}\right|\right\}$. Here the max is defined to be 0 if all numbers in the set are $<0$. Because of (3), $\left\{\alpha^{i} \mid i<\omega\right\}$ is linearly independent and we have

(5) $R / I \cong\left(J_{p}[\alpha]\right)^{\wedge}$, the $p$-adic completion of the polynomial ring $J_{p}[\alpha]$.

This implies

(6) $R /(p R+I) \cong G F(p)[\alpha]$, the polynomial ring over $G F(p)$.

This implies $p R \subset J(R) \subset p R+I$.

(7) Let $j<k+1$. Then $\left|p^{j} f_{k} x\right|>j=\left|p^{j} f_{k}\right|$ for all $x \in I$, the ideal generated by $\beta$ and $\gamma$, i.e. $I \subset H(\bar{B})$.

This follows from a straightforward computation using that $B$ is $\Sigma$-cyclic. Observe that $I^{2}=0$.

(8) $J(R)=p R+I$.

We want $R$ to be pure in $E(\bar{B}), \bar{B}$ the torsion-completion of $B$. We show a little more:

(9) $R \oplus E_{s}(\bar{B})$ is pure in $E(\bar{B})$. (One needs this to do a "Black Box" construction; cf. [CG].)

To prove (9), let $\varphi \in E(\bar{B}), r \in R, \sigma \in E_{s}(\bar{B})$ and $n \in \omega$ with $p^{n} \varphi=r+\sigma$. Since $\sigma$ is small, there is $k \in \omega$ such that $p^{k-n-1} f_{k} p^{n} \varphi=p^{k-n-1} f_{k} r$ and $p^{2} p^{k-1} f_{k} \varphi=0$. Hence $p^{k-n+1} f_{k} r=0$. Now let $r$ be represented as in (3) and apply (4) to obtain:

$$
k-n+1 \geq \exp \left(f_{k} r\right)=\max _{i \in \omega}\left\{k+1-\left|a_{i}\right|, k+1-\left|b_{i}\right|, k+1-\left|c_{i}\right|\right\} .
$$

This implies $k-n+1 \geq k+1-\left|a_{i}\right|$ and $\left|a_{i}\right| \geq n$. The same holds for the $b_{i}$ 's and $c_{i}$ 's. Therefore $r \in p^{n} R$ and $r=p^{n} s$ for some $s \in R$. Thus $p^{n}(\varphi-s)=\sigma$ is small which implies $\varphi-s$ is small and $\varphi \in R \oplus E_{s}(A)$.

(10) Let $A$ be a pure subgroup of $\bar{B}$ containing $B$ and $\varphi \in H_{E(A)}(A, B[p])$. Then $\varphi \in H_{E(A)}(A, A[p])$.

Let $a \in A[p]-B[p]$. Since $A / B$ is divisible, there is $b \in B, y \in A$ such that $a=b+p^{n+1} y$ where $n=|a|$. Then $|a|=|b|$ and $|a \varphi|=\left|\left(b+p^{n+1} y\right) \varphi\right| \geq n+1>$ $n=|a|$ since $\varphi \in H_{E(A)}(A, B[p])$. This inequality shows $\varphi \in H_{E(A)}(A, A[p])$.

Now we apply A. L. S. Corner's result [C, Theorem 2.1] and obtain a pure subgroup $A$ of $\bar{B}$ containing $B$ and $\operatorname{End}(A)=R \oplus E_{s}(A)$. (Observe that (4) implies that condition (C) of [C, Theorem 2.1] holds.) The ring $R$ is constructed to satisfy $H_{R}(A)=p R+\alpha R+R \gamma$. Moreover we have $\alpha \in h_{R}(A)-J(R), \beta \in J(R)-H_{R}(A)$ and $\gamma \in H_{J(R)}(A)-p R$. Observe that $\gamma \notin C(A)$, since otherwise $\bar{B}[p] \gamma \subset A$. In order to see that this is absurd, we have to look into Corner's proof $[\mathbf{C}]$ of his Theorem 2.1: Recall that for a positive integer $e$ an element $x \in \bar{B}$ is $e$-strong if $x r=0$ implies $r \in p^{e} R$ for $x \in \bar{B}\left[p^{e}\right]$ and $r \in R$. Our group $A$ is one of Corner's $G_{\rho}$ (cf. [C, Theorem 2.1]). Corner observes [C, p. 285, line -6] that each of his $G_{\rho}$ contains for any $e$ an $e$-strong element $x$ such that for $\sigma \neq \rho$ we have $G_{\sigma} \cap x R=0$. For $e=1, x \in \bar{B}[p]$ and since $\gamma \notin p R$ we conclude $x \gamma \neq 0$. This shows that $\bar{B}[p] \gamma$ is not contained in any $G_{\sigma}(=A)$. Now Theorem 1 applies and we have that $J(\operatorname{End}(A))=H_{J(R)}(A) \oplus H_{E_{s}(A)}(A)$ is not contained in $C(A) \cap H(A)$ since $\gamma$ is not and also $H_{R}(A)$ is not contained in $J(R)$. So if we want to describe the elements of $J(\operatorname{End}(A))$ by their action on $A$, we have to find the elements in $J(R) \cap H(A)$, which means we must be able to recognize the elements of $J(R)$. There is much freedom for the way an element of $J(R)$ can operate on $A$. We answer a question 
in $[\mathbf{S}]$ by summarizing part of our discussion in

THEOREM 2. There exists a separable p-group $A$ such that $J(\operatorname{End}(A))$ is larger than $H(A) \cap C(A)$.

REMARK. If we want to have larger groups $A$ realizing $R$, we may employ Shelah's "Black Box" and a construction very similar to the one in $[\mathbf{C G}]$. We would like to mention again that all the $p$-groups constructed in $[$ CG, DG1 or DG2] satisfy $H(A) \cap J(R)=p R$.

\section{REFERENCES}

[C] A. L. S. Corner, On endomorphism rings of primary abelian groups, Quart. J. Math. Oxford 20 (1969), 277-296.

[CG] A. L. S. Corner and R. Göbel, Prescribing endomorphism algebras, a unified treatment, Proc. London Math. Soc. 50 (1985), 447-479.

[DG 1] M. Dugas and R. Göbel, On endomorphism rings of primary abelian groups, Math. Ann. 261 (1982), 359-385.

[DG2] _ Almost $\Sigma$-cycle abelian p-groups, Proc. Udine Conf. on Abelian Groups and Modules, Springer-Verlag, Wien and New York, 1984, pp. 87-106.

[F] L. Fuchs, Infinite Abelian groups, Vols. I and II, Academic Press, New York and London, 1973.

[H] J. Hausen, Quasi regular ideals of some endomorphism rings, Illinois J. Math. 21 (1977), 845-' 851.

[HJ] J. Hausen and J. A. Johnson, Ideals and radicals of some endomorphism rings, Pacific J. Math. 74 (1978), 365-372.

[L] W. Liebert, The Jacobson radical of some endomorphism rings, J. Reine Angew. Math. 262 (1973), 166-171.

[P] R. S. Pierce, Homomorphisms of primary abelian groups, Topics in Abelian Groups (J. Irwin and E. Walker, eds.), Scott, Foresman and Co., Chicago, Ill., 1963.

[S] A. D. Sands, On the radical of the endomorphism ring of a primary abelian group, Proc. Udine Conf. on Abelian Groups and Modules, Springer-Verlag, Wien and New York, 1984, pp. 305-314.

Department of Mathematics, Baylor University, Waco, Texas 76798 